Pus, then, has no peculiar or specific action when present in the blood. If putrid, it will act like putrid matters generally do ; or its globules may, under certain circumstances, like other foreign particles, become arrested in and block up the capil. laries, producing effects already described.

The clinical relation of pus to the production of pyæmia appears to be this :-

Suppuration is a common occurrence, either on the surface of wounds or ulcers, or in internal parts.

Pus, as pus, cannot be absorbed ; that is, its solid particles, its corpuscles, cannot enter the circulation, except through some lesion of the bloodvessels. Pus may pass into the circulation through an orifice in a vein ; this occurrence, however, must be extremely rare. But the fluid part of pus may be absorbed. If this be not putrid no mischief will supervene.

Even if healthy pus should, by some rare chance, find its way into the circulation, no secondary abscesses would perhaps be, under ordinary circumstances, produced. For healthy pus may be, very often has been, injected into the circulation with out being followed by any purulent deposit whatever. None of the effects which are ascribed to pyæmia are necessarily produced by the injection of healthy pus.

But pus may become putrid, more especially if exposed to air in a wound, and the putrid fluid may be absorbed; and this, like any other putrid fluid, will produce the effects to which the term pyæmia is applied. It will poison the blood. For when putrid pus is injected the symptoms of blood-poisoning as after the injection of any putrid matter, will supervene.

It is undoubtedly a rare circumstance to find a case of wellmarked and fully developed pyæmia without any evidence of the previous existence of pus in some part of the body. The previous existence of an external wound, indeed, is by no means necessary to the development of pyæmia. In children, on the contrary, it is perhaps most frequently seen in connexion with acute necrosis. But suppuration occurs in these cases.

Now one of the chief arguments of those who affirm that the passage of pus into the blood is the sole cause of pyæmia is drawn from the assumed fact of the constant pre-existence of a local suppuration. But the fact that pyæmia is so commonly preceded by the formation of pus admits of another, which I believe is the correct interpretation.

Pyæmia is due to the absorption of a putrid or morbid fluid. But such a fluid is hardly ever, can hardly ever be, poured out except under the circumstances in which pus will be formed. Nay, more; no doubt this morbid, poisonous fluid is often the changed or partially decomposed fluid of pus. The conditions under which suppuration occurs are almost always the conditions under which a putrid fluid will be formed and can be absorbed. Admitting fully, however, this relation of pus to pyæmia, it is nevertheless to be understood that there is nothing peculiar to pus upon which the production of pyrmia depends.

When one reflects upon the subject, it is not perhaps so difficult as at first sight it may appear to be to account for the fact that the same effects are produced by such different agents as poisonous fluids and passive particles of solid matter.

To take the second case first. It cannot be doubted that these minute particles act mechanically only, at least at the outset, by blocking up capillary vessels, and so inducing stagnation and congestion, which gradually spread; the farther consequences being determined by other circumstances, such as the extent of irritation thus set up, and the constitution and state of health of the subject.

In the first case also, stagnation is likewise induced, but by a different process. It is essential to a free capillary circulation that the changes between the blood and the tissues which there occur should proceed in a normal manner. It has been shown that whatever interferes with those mutual changes constitutes an impediment to the capillary circulation. Hence, then, the congestion and stagnation, more or less extensive and widely spreading, which ensue upon, or can be induced at pleasure by, the introduction of morbid matters into the blood. These may be altogether in the fluid form, yet, mingling with the blood, they must affect profoundly its constitution, and consequently the relation which it will hold to the tissues through which it circulates. Healthy natrition is checked the normal changes are obstructed, and, therefore, the capil lary circulation is no longer free. The transmission of blood through the capillaries being hindered, it there accumulates, and at length stagnates. Thus obstruction to the circulation of the blood through the capillaries is the common condition arrived at in either case.

It is a significant fact that these different agents not only produce the same result, but affect principally and particularly the same organs-the lungs. In the case of solid particles, which obviously act by blocking up the capillaries, the lungs are the sole or chief seat of the mischief, by virtue of their position-their capillaries being the first that the blood reaches after its contamination, - their vascularity, and the minuteness f their capillary network. What other explanation, in the ase of morbid tluids, can be offered of the fact, that here also the stress of mischief falls upon the lungs, than that the cause of it is likewise lodged in the pulmonary capillaries? In either case the organs beyond are more or less protected by the total r partial arrest of the mischievous matter in the lungs.

It may, perhaps, be said that, generally speaking, the spots of stagnation and congestion produced by the injection of solid particles are smaller and more regular in outline than the patches produced by the injection of poisonous fluids. This variation, which constitutes of course no essential distinction, may be due to the fact that a fluid is more readily and widely diffused through portions of the blood than are solid particles.

It must be observed that the effects produced by the introduction of different matters into the blood is by no means uniform. They may differ widely. Thus, after the mingling of certain poisonous substances with the blood, instead of suppuration, gangrene of the lungs and other organs may be produced. This fact seems to be well established both by experiment and by observation. But does this result-gangrenerepresent a difference of kind, or only of degree? Are sup. puration and gangrene-to take these striking results-distinct effects of different poisons?-or are they only different degrees of morbid action, determined by the intensity or quantity of the poison operating, and the condition of the system? All I can say is this : that the evidence before us does not warrant the conclusion that these effects are due to the action of different poisons.

The causes of the local congestions and suppurations in pyæmia may, therefore, be thus classified.

Stasis due to mechanical action: a blockade produced by the impaction of solid particles. According to their size-

Arterial embolism: by fragments too large to pass through the smaller arteries.

Capillary embolism: by fragments small enough to pass into the capillaries.

Stasis due to change in the blood produced by the admixture of morbid fluid: the local effect of blood-poisoning, properly so-called-

Capillary obstruction.

Stasis due to a combination of the two above-mentioned causes.

In all cases stagnation and congestion first ensue. The subsequent changes, whether towards resolution, suppuration, or gangrene, are determined by-

The action of the morbid fluid or obstructing substance. The changes it provokes.

The constitution and state of health of the individual.

(To be continued.)

\section{ON STRICTURE OF THE CERVICAL CANAL.}

BY E. J. TILT, M.D., M.R.C.P.,

CONSULTING PHYSICIAN TO THE FARRINGDON DISPENSARY

(Concluded from vol.ii. 1866, p. 631.)

From what has previously been stated it will be obvious that it is an imperfect diagnosis that leads surgeons to divide the cervix so much more frequently than is warranted by sound pathology. Dysmenorrhœa is so frequently caused by uterine disease, that we must not think of attributing it to physical obstruction to the passage of the menses, until inflammation and congestion have been removed by depleting the womb, by vaginal injections, and by the use of agents which, like nitrate of silver, substitute healthy for diseased nutrition of tissue. Many a case of dysmenorrhoea, supposed to be obstructive, will yield to persistence in the intelligent use of these measures. and I am quite ready to admit Dr. Routh's assertion that hypertrophy and chronic congestion of the mucous membrane lining the cervix may be sometimes beneficially modified by what I should call the internal scarification of the cervical canal.

When all trace of inflammation has been removed it is time 
to infer that dysmenorrhœa depends upon some obstruction to the free passage of the menses, and this can only be ascertained by probing the womb. While giving to Sir James Simpson all credit for having taught us how to sound the womb, and the value of the information to be thus obtained, I agree with Dr. Marion Sims that it is better to use as little as possible the instrument sold as his; and if I have stated that I consider it unjustifiable to slit the neck of the womb when the uterine sound could be passed without difficulty, my object was to express a means of measurement that everybody could understand-a precaution rendered necessary by the fact, that those who recommend the frequent performance of this operation omit to state what they consider to be the normal width of the cervical canal.

When a resistant probe is required, I prefer Chassaignac's uterine sound, which is shorter than Simpson's, and only slightly curved at its extremity ; but for ordinary use there is nothing better than the well-known wax bougies: they seem to have a peculiar aptitude to pass through the strictured canals, and I have often been able to pass one when $I$ have failed with the uterine sound, the calibre of the two instruments being the same. Another great advantage of using wax bougies is, that it is difficult to do harm with them. If they cannot pass the stricture, they bend and are expelled by the womb, whereas the uterine sound often canses great pain and loss of blood, sometimes inflames the womb or the surrounding tissues, and has been repeatedly known to perforate the womb when used by inexperienced hands. No one should introduce a bougie, and still less a metallic sound, in to the womb, without previously ascertaining its shape and position, so as to know what curve it may be useful to give the probe, and in what direction to introduce it. It must be particularly ascertained, by means of the finger in the vagina and sometimes in the rectum, whether the body of the womb is not fiexed upon the cervix; for those who do not detect this condition will of course find great difficulty in passing the sound, or may not be able to do so, and may subject their patients to useless treatment by sponge tents, as I have seen in several instances. It will be obvious that in cases of this kind the wax bougies are of much safer use than the rigid metal rod, and if the wax bougie be carefully brought away, it will, to a certain extent, bear the impression of the curve in which it has been moulded.

With regard to the dangers attending the division of the cervix, they will depend upon the extent to which the division is carried. If the cervix has been divided right and left, down to its vaginal attachments, one cannot be too careful of the patient for the following days ; but this is very seldom required. In the majority of cases in which one or two longitudinal incisions, to the depth of two or three lines, are made inside the cervical canal, particularly when the os internum is not divided, or when the os externum and adjacent tissues are thoroughly divided to the extent of three-quarters of an inch, it is quite unnecessary to subject the patient to the fuss and expense of a skilled nurse, or to enforce silence, and keep "the patient in bed for three weeks, as if some fearful operation had been performed. Common sense will teach the happy medium between foolhardiness and a ridiculons amount of precaution, and I am desirous of saying this, for I have myself exaggerated the dangers of the operation in my "Handbook of Uterine Therapentics."

Surgical skill is the highest kind of skilled labour, and the surgeon has the right to value his work as he likes. The public need not employ him if they think he overestimates his skill; nevertheless there is a tacit understanding with respect to the fees that should be asked for various operations, and surely there should be a similar agreement respecting the fee for division of the cervix. At present some practitioners take five or ten guineas, which seems to me too moderate a fee, while others ask a hundred or a hundred and twenty five guineas, which is obviously exorbitant, considering that no more is asked for ovariotomy, or for amputation of the thigh.

Grosvenor-street, Grosvenor-square, Jan. 1867.

Scurvy. - We find by returns received from Liverpool, and collected by Mr. Reginald Harrison, Lecturer on Anatomy at the Liverpool School of Medicine, that the number of cases admitted into hospitals in that town during the past year exceeds by fifteen thoze entered on the books of the Dreadnought during the corresponding period. The Royal Infirmary furnishes a list of 22 , the Northern Hospital of 35, and the Southern Hospital of 59 cases, out of which 2 deaths have occurred at the Southern Hospital and 1 at the Royal Infirmary.
REMOVAL OF A METALLIC PENCIL-CASE FROM THE BLADDER OF A WOMAN SIX DAYS AFTER ITS INTRODUCTION.

\author{
WITH INTRODUCTORY CLINICAL REMARKS.
}

BY THOMAS NUNNELEY, F.R.C.S., SURGEON TO THB LEEDS GENERAI INEIKMARY.

GentLemer,-Following the course which, as a rule, I have adopted in my clinical lectures, of calling your attention to those cases under my care in our General Infirmary which are of great interest and importance, whether from the rarity of the affection, the importance of the disease, the obscurity of its diagnosis, the difficulty of its treatment, its being a typical illustra. tion of a particular affection, its being a connecting link between disorders at first sight not presenting much in commor with each other-as the very varied forms of erysipeloid affections, which some of you will recollect I have more than once brought under your notice, with the view of proving to you how varied and, apparently, isolated complaints, having individual names and histories, are really but varied forms of this protean malady, owning a common origin and interchangeable, each form capable of inducing the other forms ; or even of complaints of more frequent occurrence and of more easy management, but of which the importance of knowing them well is in your future career certain to be great; - I shall call your attention to the case of a woman whom many of you have lately seen in No. 10 ward. Though it is certain none of you will frequently be called upon to treat such a case, and even probable that many of you may not meet with a single one, yet it is of great importance when it does occur that you should understand it, and of interest that you should be prepared to manage it with skill and decision, or the consequence may be very serious to your patient.

This, as $I$ understand the term, is the true meaning and value of "clinical lecture," rather than the delivery of an essay on the principles and practice of surgery. These, I hold, should rather be learned in the course on the principles and practice of surgery, or by private study of those excellent books and dictionaries on surgical science which it is open to all to obtain; or, better still, by a careful reading of original essays by writers who have made the particular subject their especial study. Bedside lectures, I think, are more appropriately devoted to pointing out from the patient then directly under observation the meaning and value of symptoms there presented to us, the inferences which are to be deduced from them, the treatment which is most applicable, and the reasons why one remedy or one procedure is adopted in that particular case rather than another; for though we may follow, in all cases of the same disease, the same general outline of treat. ment, yet we shall find in each case, whether arising from constitution, age, or idiosyncrasy, the stage of the complaint, the particular part where it happens to be located, or any other cause-and there may be many, - that great and important modifications are needed. Now, the proper adaptation of these modifications is all-important, and, not unfrequently, makes all the difference between curing the patient, and, if not absolutely injuring him, at any rate not doing him any good. In fact, it is this knowledge, which experience and tact confer upon the well-grounded and thonghtful practitioner, which is so valuable to his patient, and which distinguishes the man of experience from the theorist and merely erudite man. This is nowhere to be so well acquired as at the bedside of patients under the guidance of those who are com. petent to the task. It makes no difference to the character of the lecture whether given in this theatre, at the school, away from the infirmary, or by the bedside of the patient. This place is merely chosen as a matter of great convenience, not only to the patient who is the immediate subject of the remarks, but also to you ; for symptoms, treatment, and results can all be more freely discussed away from him than they possibly can be in his anxious hearing.

You will therefore understand that I expect you all to have some acquaintance with the principles of surgery. It is my object to point out to you, as cases arise, how you may best apply these principles to the particular case which is the sub- 